This item was submitted to Loughborough's Research Repository by the author.

Items in Figshare are protected by copyright, with all rights reserved, unless otherwise indicated.

\title{
Ultrasonic measurements and machine learning for monitoring the removal of surface fouling during clean-in-place processes
}

PLEASE CITE THE PUBLISHED VERSION

https://doi.org/10.1016/j.fbp.2020.05.003

\section{PUBLISHER}

Elsevier B.V. on behalf of Institution of Chemical Engineers

VERSION

AM (Accepted Manuscript)

\section{PUBLISHER STATEMENT}

This paper was accepted for publication in the journal Food and Bioproducts Processing and the definitive published version is available at https://doi.org/10.1016/j.fbp.2020.05.003.

LICENCE

CC BY-NC-ND 4.0

\section{REPOSITORY RECORD}

Escrig-Escrig, Josep, Alessandro Simeone, Elliot Woolley, Shreedhar Rangappa, A Rady, and Nicholas Watson. 2020. "Ultrasonic Measurements and Machine Learning for Monitoring the Removal of Surface Fouling During Clean-in-place Processes". Loughborough University. https://hdl.handle.net/2134/12264518.v1. 


\title{
Ultrasonic Measurements and Machine Learning for Monitoring the Removal of
}

\section{Surface Fouling during Clean-in-Place Processes}

\author{
J. Escrig, Escrig ${ }^{1,2}$, A. Simeone ${ }^{3}$, E. Woolley ${ }^{4}$, S. Rangappa ${ }^{4}$, A. Rady ${ }^{1}$ \& N. J. Watson ${ }^{1 *}$
}

${ }^{1}$ Food, Water, Waste Research Group, Faculty of Engineering, University of Nottingham, University Park, Nottingham, NG7 2RD, UK

${ }^{2}$ i2CAT Foundation, Calle Gran Capita, 2 -4 Edifici Nexus (Campus Nord Upc), 08034, Barcelona, Spain

${ }^{3}$ Intelligent Manufacturing Key Laboratory of Ministry of Education, Shantou University, Shantou 515063, China

${ }^{4}$ Wolfson School of Mechanical, Electrical and Manufacturing Engineering, Loughborough University, Loughborough, LE11 3TU, UK

${ }^{*}$ Corresponding Author.

Address: Dr Nicholas James Watson. Room B16, Coates Building, University Park, University of Nottingham, Nottingham, Nottinghamshire, NG7 2RD, UK.

Email: nicholas.watson@nottingham.ac.uk

Phone: (+44) 1157484848

\section{Abstract:}

Cleaning is an essential operation in the food and drink manufacturing sector, although it comes with significant economic and environmental costs. Cleaning is generally performed using autonomous Clean-in-Place (CIP) processes, which often over-clean, as suitable technologies do not exist to determine when fouling has been removed from the internal surfaces of processing equipment. This research combines ultrasonic measurements and machine learning methods to determine when fouling has been removed from a test section of pipework for a range of different food materials. The results show that the proposed methodology is successful in predicting when fouling is present on the test section with accuracies up to $99 \%$ for the range of different machine learning algorithms studied. Various aspects relating to the training data set and input data selection were studied to determine their effect on the performance of the different machine learning methods studied. It was found that the classification models performed better when data points were extracted directly from the ultrasonic waves and when data sets were combined for different fouling materials.

Key words: Ultrasonic Measurements, Machine Learning, Clean-in-Place, Industrial Digital Technologies 


\section{Introduction}

Cleaning of processing equipment is one of the most important operations in sectors such as food and drink, pharmaceutical and Fast Moving Consumer Goods (FMCG). Cleaning is performed to ensure the equipment is hygienic, the products remains safe for consumption and there is no product cross-over. Cleaning is also essential to ensure processing equipment operates at optimal performance. For example heat exchangers are known to have lower heat transfer rate as fouling layers form and grow on their internal surfaces (Wen et al., 2017). The internal cleaning of processing equipment in medium to large-scale production facilities is usually performed by an automated process, called Clean-in-Place (CIP).

Clean-in-Place systems have separate tanks and pipework to the processing equipment, which are used to hold and transport cleaning water and chemicals. The advantage of CIP systems is that they can clean the internal surfaces of processing equipment without disassembly or manual operator cleaning, thus speeding up the process. The cleaning in CIP is performed by a combination of mechanical force, time, temperature and chemicals, which are considered in the Sinner's Circle (Sinner, 1959). Most CIP systems feature multiple cleaning, rinsing and sterilisation stages with a typical CIP set being: 1) pre rinse 2) chemical cleaning 3) post rinse 4) sanitisation 5) final rinse (Fryer et al., 2006). The CIP set will be designed for a specific set of processing equipment and routinely validated using Adenosine Triphosphate (ATP) measurements or growth and microbial enumeration tests (Fratamico et al., 2009).

Cleaning processes are known to have significant negative environmental impacts due to the amount of water, energy and chemicals they use. It has been reported that cleaning is responsible for $30 \%$ of the energy used in dairy processing (Eide et al., 2003), and approximately $35 \%$ of the water used in beer production (Pettigrew et al., 2015). Cleaning operations also effect the productivity of a factory as they reduce the amount of time available for manufacturing products.

Although CIP systems are a significant improvement on manual cleaning, challenges in their design and operation remain. The main challenge is that the majority of CIP processes have no quantitative methods to determine how clean the internal surfaces are at any point in the process. Clean-in-Place processes are designed primarily with safety in mind, which results in constant over-cleaning of processing equipment. This over-cleaning is a waste of resources and time and increases the negative environmental and economic impacts of cleaning. Another significant challenge is that CIP processes are generally designed to clean one type of fouling material. This presents problems in the food and drink sector where there is a current drive to use existing processing equipment to manufacture a larger range of products to meet consumer demands. With different materials known to foul and be cleaned from processing equipment differently (Wilson, 2018), there is a need for CIP systems to be 
more adaptive in these cleaning operations to ensure all fouling material is removed whilst minimising over-cleaning.

Research activities related to the optimisation of CIP processes has focussed on understanding the effects of cleaning parameters such as temperature and water flow rate (Fan et al., 2018), or by recording simple measurements from the equipment (e.g. temperature changes) (Fratamico et al., 2009). Research has been performed studying temperature (Vieira et al., 1993) and pressure (Riverol and Napolitano, 2005) changes at different locations within the equipment during cleaning, in addition to monitoring the properties of the cleaning fluids exiting the equipment after cleaning (Lyndgaard et al., 2014) (Berg et al., 2017) (Van Asselt et al., 2002). Although monitoring the dirty cleaning water can be used to determine the presence of fouling that has entered the water it cannot directly monitor fouling which remains on the equipment's internal surfaces. As one of the primary functions of cleaning is to remove this surface fouling, research has been performed to monitor this using electrical (e.g. (Wallhäußer et al., 2012a) (Chen et al., 2003) (Guérin et al., 2007) (Tlili et al., 2008)), acoustic (e.g. (Pereira et al., 2009) (Withers, 1996) (Úbeda et al., 2016)) and optical methods (e.g. (Withers, 1996) (Simeone et al., 2018) (Cheong et al., 2017)(Simeone et al., 2016) (Tamachkiarow and Flemming, 2003)). Ultrasonic (US) techniques are a branch of acoustics which utilise mechanical waves operating in the US frequency range $(>20 \mathrm{KHz})$. Ultrasonic techniques are an ideal process sensor as they are low cost and can operate non-invasively in opaque systems (Watson, 2015). The first work utilising US techniques to monitor fouling was performed by Withers in the 1990s (Withers, 1994). This work used two US transducers to measure the fouling thickness of a range of food and non-food fouling materials in a bespoke laboratory scale rig filled with a fluid (Withers, 1994) (Withers, 1996). Since this time a range of research has been performed using US techniques to monitor fouling and cleaning ((Wallhaußer et al., 2011), (Wallhäußer et al., 2012b), (Wallhäußer et al., 2013), (Wallhäußer et al., 2014) (Úbeda et al., 2016), (Chen et al., 2019)(Escrig et al., 2019)).

Machine Learning $(\mathrm{ML})$ is an application of Artificial Intelligence that utilises predictive algorithms for classification or regression problems. Supervised ML methods such as Artificial Neural Networks (ANN) and Random Forests (RF) use a data set to train the algorithms (Goodfellow et al., 2016). Input data can come from a variety of sources including features extracted from sensor measurements and other experimental parameters (e.g. fluid temperature and flowrate). Advantages of $M L$ techniques include the volume and variety of data they can process and their ability to improve accuracy as more or better data sets become available. In addition, they do not require the development of physical inversion models, accounting for complexities such as variable temperature, and can process data quickly once the initial training stage has been completed. Research utilising ML methods within fouling and cleaning operations has been performed, with the majority of work focussing on heat exchanges. 
103

104

105

106

107

108

109

110

111

112

113

114

115

116

117

118

119

120

121

122

123

124

125

126

127

128

129

130

131

132

133

134

135

136

Research in this area has primarily used ANNs and been able to accurately predict outputs such as fouling deposit thickness, fouling resistance and optimal time remaining before the heat exchanger should be cleaned (Riverol and Napolitano, 2005), (Sun et al., 2008), (Garcia, 2012), (Mohanty and Singru, 2014) (Wen et al., 2017), (Wang et al., 2018). These reported works generally used measurements of fluid properties (e.g. temperature, flow rate, turbidity, pressure) as inputs for the developed models. Examples of non-heat exchanger applications include (Zhang et al., 1999) who developed an ANN to estimate the degree of fouling in a batch polymerization reactor using temperature and flow measurements; and Shetty and Chellam, 2003 who developed an ANN to predict the fouling in a nanofiltration membrane. They used flow rates and water quality parameters (e.g. $\mathrm{pH}$ and total suspended solids) as inputs to their model and could predict the degree of fouling with less than 5\% absolute error. Simeone et al., 2018 developed an ANN, which utilised images from an ultraviolet fluorosensing method to monitor the cleaning of a tank, representative of those used in the food industry. This work showed that the system could be used to monitor the surface coverage of fouling and fouling thickness within the tank. An ANN was used to predict the time remaining to clean the tank based on the current fouling condition. This prediction is beneficial to industry as it enables enhanced scheduling for the use of the processing and cleaning equipment.

Although ML has not seen widespread adoption for US measurements, examples exist for flow regime classification (Figueiredo et al., 2016) and non-destructive testing of pipes (Cau et al., 2005).

The combination of US measurements and ML methods to identify fouling and monitor cleaning processes has previously been studied (Wallhaußer et al., 2011), (Úbeda et al., 2016; Wallhäußer et al., 2014, 2013). These works focussed exclusively on dairy fouling and utilised two different types of ML approaches: ANN and Support Vector Machines (SVM). The initial work from this group focussed on measurements of a fouling layer in a static system. Wallhaußer et al., 2011 transmitted acoustic waves through a test cell with a fouling layer. They recorded acoustic reflections with an US transducer attached to an external surface of the test cell. They calculated the acoustic impedance and the echo energy from the reflected waves, which they used as inputs for an ANN. They showed that this approach could predict the presence of fouling with an accuracy of 99\%. Wallhäußer et al., 2013 extended this work by studying the two different components of dairy fouling separately and investigating the use of SVM in addition to ANN. They found that the protein fouling was much easier to identify using their method than mineral fouling (ANN accuracy $100 \%$ compared to $93.5 \%$ ). They found that SVM models gave much higher prediction accuracy than ANN, and reported that the ML models performed much better when trained just on the fouling material (mineral or protein) for which they would predict fouling. The group continued this work to monitor the actual cleaning of fouled surfaces under flow conditions (Úbeda et al., 2016; Wallhäußer et al., 2014). They showed that 
137

138

139

140

141

142

143

144

145

146

147

148

149

150

151

152

153

154

155

156

157

158

159

160

161

162

163

164

165

the combination of features extracted from reflected US signal could be used as inputs in ML models to predict the presence of fouling. They found that SVM methods performed better than ANN, and that the accuracy of the models could be improved by including other features such as temperature (known to affect US wave propagation). Although work has been performed using ML and US sensors to monitor cleaning this has focussed exclusively on dairy fouling and has not investigated many other factors related to the model development and training.

This current work investigates the use of ultrasonic measurements and a range of different classification ML methods to predict when cleaning will be complete for a range of different fouling materials relevant to the food and drink sectors. A bespoke transparent laboratory scale rig was used and images of the cleaning processes were recorded to enable the training of the ML models. This work studies how the performance of the machine learning models is affected by variables such as input data selection method and training data set construction.

\section{Materials and Methods}

\subsection{Experimental data collection}

Figure 1 depicts the experimental rig which was designed and built for the laboratory scale experiments. The rig was a rectangular section with a $1.2 \mathrm{~mm}$ thickness stainless steel (SS 430 ) bottom plate. The sides and top of the rig were made of Perspex such that images of the fouling removal could be recorded. The rig was $300 \mathrm{~mm}$ long with an internal width and height of $40 \mathrm{~mm}$. Although the majority of industrial cleaning features pipework of a circular cross-sectional area, in this work a rectangular rig was developed in order to: 1) aid the imagining of the fouling removal, and 2) eliminate the complexities associated with attaching the US transducer to a curved surface. Valve controlling mains water pressure were located at either end of the rig to allow fluids to flow through and perform the cleaning.

Camera

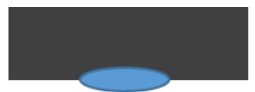

Transparent Wall

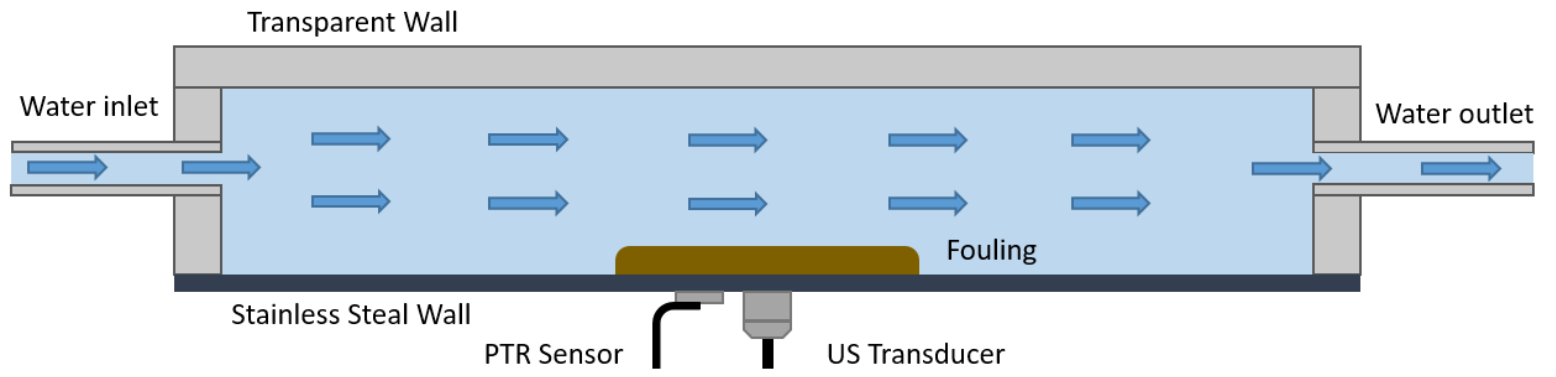

Figure 1: Laboratory setup including sensor and fouling material locations and direction of fluid flow. 


\subsubsection{Instrumentation}

The experimental rig was fitted with an US transducer, a camera and a temperature sensor to collect data during the cleaning experiments. The location of the instruments can be seen in Figure 1 . The US transducer was a $5 \mathrm{MHz}$ magnetic contact transducer (Olympus ${ }^{\circledR}$ ). This was attached to the bottom of the rig, a thin film of couplant fluid was placed between the US transducer and the SS430 wall to enable transmission of US waves into the material. The US transducer was connected to a US box (Lecoeur Electronique ${ }^{\circledR}$ ), which was utilised to excite the US transducer and digitise the received US waves. The camera utilised was a Logitech ${ }^{\circledR} \mathrm{C} 2703 \mathrm{MP}$ web camera. Temperature was recorded using a RTD PT100 which was attached to a Pico Technology ${ }^{\circledR}$ PT-104 data logger. The US box, PT-104 data logger and web camera were all connected to a laptop. Instrumentation control and data collection were all performed via bespoke software developed in MATLAB ${ }^{\circledR}$. During the experiments, US and temperature data was recorded every 4 seconds and Images from the camera were recorded every 20 seconds.

\subsubsection{Food Fouling Materials}

Tomato paste, gravy and concentrated malt extract were used as the fouling materials. Three different food materials were selected as it has been reported that different materials foul, and are cleaned from surfaces differently (Fryer and Asteriadou, 2009) (Cuckston et al., 2019). Therefore it is important to perform measurements on a range of different fouling materials to determine the capabilities of the US sensor and ML methods for different industrially relevant materials. The tomato paste was Napolina ${ }^{\circledR}$ Double Concentrate Tomato Pure and the ingredients were: tomatoes, acidity regulator (citric acid). The gravy was Bisto ${ }^{\circledR}$ Favourite Gravy Granules with the following ingredients: potato starch, maltodextrin, palm oil, salt, wheat flour, colour (E150c), sugar, flavour enhancer (E621, E635), emulsifier (E322). The concentrate malt was taken from a Coopers ${ }^{\circledR}$ Real Ale beer kit with the following ingredients: malted barley, hops, yeast and water.

The gravy was prepared by mixing 10 grams of granules into $10 \mathrm{ml}$ water which was at an initial temperature of $70^{\circ} \mathrm{C}$. The water was heated in a glass beaker placed on a hot plate. Stirring was performed manually and continuously for 1 min using a metal spatula. The gravy fouling layer was created by depositing $15 \mathrm{~g}$ of the material on to the centre of the bottom plate of the rig. The fouling was placed so that the centre was approximately in the same location as the US transducer. The fouling was then spread evenly with a spatula to form a uniform layer of approximately $5 \mathrm{~mm}$ thickness. The fouling was left to cool for ten minutes before beginning the cleaning experiments. It was observed that the layers did not dry out during this time and the thickness was still approximately $5 \mathrm{~mm}$ when the cleaning experiments began. For repeat experiments a new mixture was prepared using the methodology above to ensure its properties were as similar as possible for each repeat. For 
the malt and tomato paste no preparation was required and $15 \mathrm{~g}$ of each material were applied to the surface using the spatula, again taking care to ensure they were in the same location for each experiment. For the malt and tomato paste the cleaning experiments were initiated within one minute of applying the layer as it was observed that any delays would results in the layer spreading. This spreading was not observed for the gravy as it had a higher viscosity. Although every care was taken to ensure consistency between repeat experiments it was not always possible to place the fouling in precisely the same location or ensure a layer of consistent $5 \mathrm{~mm}$ thickness. This was not considered critical to the current work as the primary focus was to assess the performance of the US sensor and ML models.

\subsubsection{Cleaning experiments}

Cleaning experiments were performed in two batches. The first to collect data to train the ML models (training data set), and the second to test the performance of these models (test data set). Cleaning was performed with water at two different temperatures $\left(12^{\circ} \mathrm{C}\right.$ and $\left.45^{\circ} \mathrm{C}\right)$ for each of the three fouling materials. Repeat experiments for each material and temperature were performed with details of the training data set (TR) in Table 1 and test data (TE) set in Table 2. In these tables the Reynolds number of the flow was calculated using the fluid velocity, the width of the rig and the kinematic viscosity of water at the experimental temperature. In general if the Reynolds number is below 2300 laminar flow is present and if it is above 2900 turbulent flow is present. However, in the current work the flow at the fouling area would not be considered fully developed as a fully developed flow is only formed at a distance of approximately the characteristic length of the section (in this case width, $40 \mathrm{~mm}$ ) multiplied by 20 . The details of the fluid flow at the fouling removal location should be taken into account if using the results in this current work to understand the cleaning mechanism of different fouling materials. For many of the experiments with gravy at the lower temperature the fouling was not completely removed, so only two repeats were possible. For the tomato paste at $45^{\circ} \mathrm{C}$ the fouling was removed too quickly so the flow rate was reduced. To begin the experiments the inlet valve on the rig was opened and it was filled slowly with water at the desired temperature. The rig was then left in a static flooded condition for the temperature to stabilise. Data collection was then initiated and the outlet tap was opened. Water was then allowed to flow through the rig whilst the fouling material was removed. Each experiment continued for several minutes after the fouling appeared to be removed from the camera images.

Table 1: Experimental variables and values for the training data set

\begin{tabular}{|c|c|c|c|c|c|c|c|}
\hline $\begin{array}{c}\text { Experiment } \\
\text { number }\end{array}$ & Material & $\begin{array}{c}\text { Temperature } \\
\left({ }^{\circ} \mathrm{C}\right)\end{array}$ & $\begin{array}{c}\text { Flow } \\
\text { rate } \\
(\mathrm{I} / \mathrm{min})\end{array}$ & $\begin{array}{c}\text { Fluid } \\
\text { velocity } \\
(\mathrm{m} / \mathrm{s})\end{array}$ & $\begin{array}{c}\text { Reynolds } \\
\text { number }\end{array}$ & Repetitions & $\begin{array}{c}\text { Number of } \\
\text { recorded } \\
\text { US waves }\end{array}$ \\
\hline TR1 & Tomato & 12 & 6 & 0.0625 & 2529 & 5 & $5 \times 200$ \\
\hline
\end{tabular}




\begin{tabular}{|c|c|c|c|c|c|c|c|}
\hline TR2 & Tomato & 45 & 1.2 & 0.0125 & 1036 & 5 & $5 \times 200$ \\
\hline TR3 & Gravy & 12 & 6 & 0.0625 & 2529 & 2 & 600,1500 \\
\hline TR4 & Gravy & 45 & 6 & 0.0625 & 5182 & 5 & $\begin{array}{c}200,300, \\
3 \times 400\end{array}$ \\
\hline TR5 & Malt & 12 & 6 & 0.0625 & 2529 & 5 & $5 \times 200$ \\
\hline TR6 & Malt & 45 & 6 & 0.0625 & 5182 & 5 & $5 \times 200$ \\
\hline
\end{tabular}

Table 2: Experimental variables and values for the test data set

\begin{tabular}{|c|c|c|c|c|c|c|c|}
\hline $\begin{array}{c}\text { Experiment } \\
\text { number }\end{array}$ & Material & $\begin{array}{c}\text { Temperature } \\
\left({ }^{\circ} \mathrm{C}\right)\end{array}$ & $\begin{array}{c}\text { Flow } \\
\text { rate } \\
(\mathrm{I} / \mathrm{min})\end{array}$ & $\begin{array}{c}\text { Fluid } \\
\text { velocity } \\
(\mathrm{m} / \mathrm{s})\end{array}$ & $\begin{array}{c}\text { Reynolds } \\
\text { number }\end{array}$ & Repetitions & $\begin{array}{c}\text { Number of } \\
\text { recorded } \\
\text { US waves }\end{array}$ \\
\hline TE1 & Tomato & 12 & 6 & 0.0625 & 2529 & 2 & $2 \times 200$ \\
\hline TE2 & Tomato & 45 & 1.2 & 0.0125 & 1036 & 2 & $2 \times 200$ \\
\hline TE3 & Gravy & 45 & 6 & 0.0625 & 5182 & 2 & $2 \times 200$ \\
\hline TE4 & Malt & 12 & 6 & 0.0625 & 2529 & 2 & $2 \times 200$ \\
\hline TE5 & Malt & 45 & 6 & 0.0625 & 5182 & 2 & $2 \times 200$ \\
\hline
\end{tabular}

\subsection{Ultrasonic data collection}

\subsubsection{Ultrasonic wave propagation in cleaning rig}

This work used a single US transducer operating in reflection mode. This is similar to the setup utilised by (Al-Aufi et al., 2019). The US transdcuer was excited by a 200 volt square top pulse generated by the US box. The received signals were amplified by $18 \mathrm{~dB}$ and digitised at a sampling rate of $160 \mathrm{MHz}$. Once an US wave was generated in the transducer it would propagate through the the SS430 plate and become incident with the plate/fouling layer interface. At this interface a proprtion of the wave would be reflected and the remaining transmitted into the fouling layer and then fluid (providing the acoustic impedance mismatch between the fouling and fluid was low enough). The proportion of reflected wave is dependent on the differences in acoustic impedance between the two materials at the interface (SS430 and fouling material or water). All reflected waves were then receievd by the same US transducer. One challenge when using US methods in thin plates, such as the experiment rig utilised in this work, is that the reflected signal may return to the transducer before the transmitted signal has fully left, resulting in overlapped waves. This occurs when the thickess of the material is less than the value specified by: $N v / 2 f$, where $N$ is the number of wave cycles in the ultrasonic pulse, $v$ is the speed of ultrasonic wave in the wall material and $f$ is the frequency of ultrasonic wave. For the US system used in this work it was found that the US waves overlapped. Although the majority of previous work utilising US to monitor fouling used wall materials with sufficient thickness to separate reflected US signals in time (e.g. (Wallhäußer et al., 2011)) it is still possible to analyse US waves were overlapping has occurred (Chen et al., 2019). The ability of US methods to operate on thin walled materials is important if the techniques are to experience an increased use within industrial environments. A large proportion of pipe work and existing processing equipment feature thin walls $(<2 \mathrm{~mm})$ and manufactures would be reluctant to replace these for the sole purpose of enabling 
measurement techniques. Ultrasonic devices are often used to perform or enhanced cleaning processes. It should be noted that the US transducer utilised in this work is a diagnostic tool, operating at a low power $\left(<10 \mathrm{~mW} / \mathrm{cm}^{2}\right)$ and would not have any effect on the cleaning process.

\subsubsection{Ultrasonic signal and data analysis}

261 An example of a reflected wave from the test rig with and without fouling can be seen in Figure 2. Although the entire waves appear to be very similar when a smaller section is viewed the differences can be seen when the different fouling materials are present (Figure $2 b$ ). All these results were taken shortly after the fouling layer was applied and so should have a similar thickness. In Figure 2 it can be seen that the received US wave is overlapped with the transmitted wave and no clear reflection echo from the plate/fouling layer interface can be identified. The signal is also saturated for the first $4 \mu \mathrm{s}$. This saturation was caused by the amplification applied to US wave. A trade-off always exists in US measurements: Increasing the transmitting voltage improves the propagation distance of the US wave and amplitude of reflected signals, but can also results in saturation.

a)

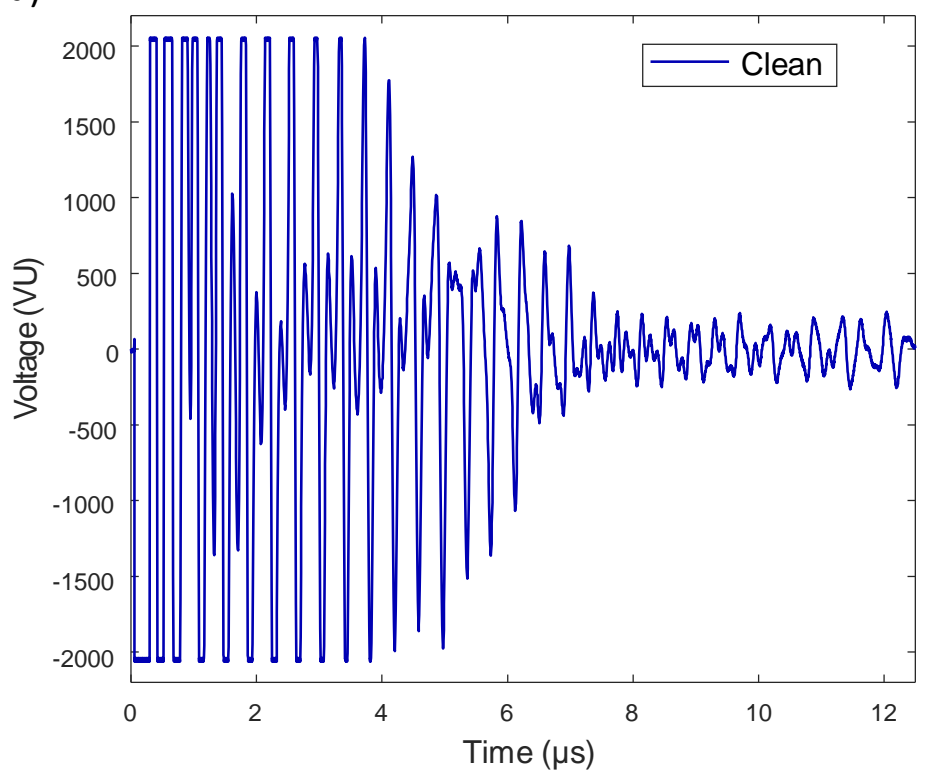

b)

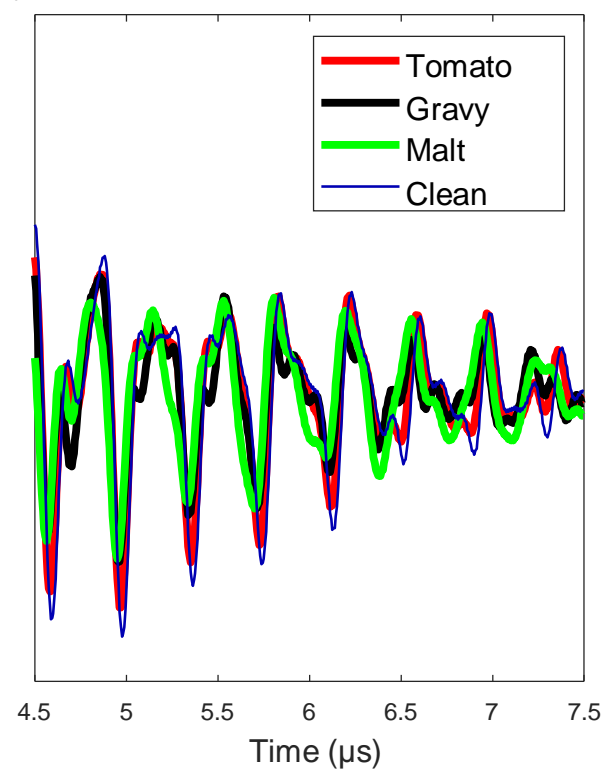

Figure 2: Example of reflected US waveforms from a) clean test section b) the presence of different fouling materials.

\subsection{Machine learning methods}

\subsubsection{Input data selection}

In this work, two different methods for determining the input data for the classification models were investigated. The first utilised data points selected directly from the US waves and the second method calculated a physical feature from the wave. By utilising two different methods it was therefore 
possible to understand the effect that the input data selection strategy has on the performance of the ML models and to determine the most reliable method.

\subsubsection{K-best predictors}

K-best predictors/features is a common methods used to determine the most suitable input data points to use for a ML model. Within this work all sampling points within a recorded waveform could be potential input data points. A signal sampling frequency of $160 \mathrm{MHz}$ was utilised for the US wave acquisition so for a 12 us recorded waveform 1920 data points were available. As data points were only taken from the non-saturated wave (4-12 us in Figure 2) this meant that a maximum of 1280 input data points were available for selection. However, some of these data points may be irrelevant or provide redundant information. In addition, a large number of data inputs increases the time required to train the models and make predictions. A large number of input data points can also result in overfitting of the model (known as the 'curse of dimensionality'). The optimal number of input data points $(\mathrm{K})$ was determined using a grid search during the validation of the models and the relevancy of each data point was determined using an F-test.

\subsubsection{Physical feature calculation}

The physical wave feature utilised as a data input point in this work was US energy. This was selected as it has been studied by previous researchers (Wallhäußer et al., 2013). To calculate the energy, a window was applied to the recorded US signals, specifically from 4 to 12 us. This window was selected as it contained no saturated signal and included the same data considered by the K-best predictors method (Section 2.3.1.1.). The energy $E(A U)$ in the windowed signal was then calculated using:

$E=\sum_{t=w_{1}}^{w_{2}} V(t)^{2}$

Where $V(t)$ is the voltage at time $t$, and $w_{1}$ and $w_{2}$ are the starting and ending times of the window respectively.

\subsubsection{Training data set}

The first round of classification used all the data for the three different materials at the two temperatures. The data was split for training and validation using the K-Fold method. This data is presented in Table 1. The test data set (Table 2) was constructed by assigning each US waveform the label of either 'clean' or 'dirty'. The US transducer has an active element area of $1.27 \mathrm{~cm}^{2}$ and is only affected by fouling on the opposite side of the plate where the transducer is located. This circular region was identified in the images taken from the camera located above the rig. A camera image was recorded every 20 seconds, during this period, five US waveforms were recorded. If any fouling was observed at the active element area of the image the five US waveforms recorded in the proceeding five seconds were labelled as 'dirty'. If no fouling was identified in the images the waveforms were 
labelled as clean. Once the active area becomes clean it would not re-foul although the precise time when it becomes 'clean' is difficult to determine as individual images were not recorded for each waveform.

\subsubsection{Classification methods}

The majority of previous research that has utilised classification methods of ultrasonic measurements to determine whether a surface contains fouling or not utilised either ANN or SVM. Although previous research utilised ANN to determine the presence of fouling for US measurements they were not studied in this work. This is due to the complexity and time required to train and optimise ANN Models. Previous research has also shown that ANN do not perform better than other classifiers, such as SVM (Wallhäußer et al., 2014).

In this work the ML algorithms used were K-Nearest Neighbour (KNN), SVM, RF and an ensemble method (adaboost). KNN classifier is a non-parametric method. The classification of a new instance has the same value as the training data points that are closer to this instance in the feature space. If $\mathrm{k}$ is equal to 1 it has the same value as the closest neighbour and if $k=n$ the instance is classified after the vote of the $n$ nearest neighbours.

Support Vector Machine is a method based on statistical learning theory aimed at determining the location of decision boundaries yielding the optimal separation of classes (Vapnik, 1999), i.e. clean and dirty in this case. For binary classification problems in which the classes are linearly separable, the SVM selects from amongst the infinite number of linear decision boundaries, the one that minimizes the generalization error. Accordingly, the selected decision boundary will be the one that leaves the greatest margin between the two classes, i.e. the sum of the distances to the hyperplane from the closest points of the two classes (Boser et al., 2004). The resulting data points closest to the hyperplane are used to measure the margin; therefore such data points are defined "support vectors" (Vapnik, 1999). In case the two classes are not linearly separable, the SVM tries to find the hyperplane that maximizes the margin while minimizing the misclassification errors. SVM can also be extended to deal with nonlinear decision surfaces, by projecting the input data onto a high-dimensional feature space using kernel functions and formulating a linear classification problem in that feature space (Boser et al., 2004).

Decision trees are a hierarchical model that can be used for classification. The instances to be classified advance through the different nodes of the tree until they finish in a labelled leaf node. The path through the nodes that they follow depends on a series of conditions that the features of the instances need to meet. The training of a decision tree classifier consists of choosing the conditions that perform the best classification of the instances. Several algorithms exist to set up these conditions (Hastie et 
al., 2009). The depth of the tree is the maximum number of splits that a path inside the tree can follow.

A deep tree can classify very accurately all the training data, but it often presents problems of overfitting when tested with new data. Another problem is that decision trees are not very robust because a small change in the data can produce a completely different tree and very different predictions.

\subsubsection{Ensemble Learning}

350

351

352

353

Ensemble learning aims at developing a population of base learners from the training data, and then combining them to form a composite prediction model. It consists of building multiple different decision tree models from a single training data set by recursively adopting multiple bootstrapped data subsets and averaging the models. Each tree is built independently to the others (Ruppert, 2004). Random forest is a bagging-based method that produces multiple trees by selecting only some of the instances of the training data and considering only some of the features of these instances to build the decisions trees. For each new tree, the instances and the features of these instances used for the training of the tree are chosen randomly. The classification of a RF is the result of the vote of the solutions given by every single decision tree. Random forests have been shown to be very robust compared to simple decision trees (Alpaydin, 2014).

Boosting is another ensemble method that refers to the problem of building a strong learner out of a collection of weak learners; i.e. learners whose predictive accuracy is only slightly better than random guessing (Sabzevari et al., 2018). Adaptive boosting (AdaBoost) is a second level ML method that combines the predictions of simple algorithms to give a final prediction. This is calculated as the weighted sum of the predictions of the first level algorithms:

$F(x)=\sum_{i=1}^{N} w_{i} f(x)$

Where $f(x)$ is the function of a simple algorithm that gives a prediction of an $\mathrm{x}$ input, $w_{i}$ is the weight of each algorithm considered in the ensemble, $N$ is the total number of algorithms used, and $F(x)$ is the final prediction. AdaBoost algorithms calculate the weights, $w_{i}$, iteratively to reduce the error of the prediction. The first level algorithms used in this work were decision trees of depth equal to 1. They were trained independently using random parts of the training data set

\subsubsection{Selection of parameters, grid search and k-fold validation}

There are several parameters of the training algorithms that need to be selected for the different classification methods. These are: the number of input data points, the maximum depth of the trees in the RF algorithms, the number of neighbours to consider in the KNN algorithm and the learning rate applied in the iteration of the AdaBoost. These parameters were optimized applying a grid search and a K-fold validation method. 
377 The number of input data points (taken from the US wave samples) introduced into the grid search 378 were values from 25 to 1200 in steps of 25 for all of the algorithms. The maximum depths of the RF 379 algorithm considered in the search were between 3 and 10 inclusive. The number of neighbours in the 380 KNN algorithm used in the validation ranged from 1 to 200 . Finally, the learning rates studied in the 381 AdaBoost were $0.5,0.75$ and 1 . The $\mathrm{k}$-fold validation was performed by dividing the training data into 3825 folds. The parameters of the grid search that scored higher in the k-fold validation were included in 383 the different models. Other parameters were not included in the grid search because adding more 384 dimensions to the grid increases the calculation time exponentially. The number of trees produced in 385 the RF and AdaBoost algorithms was set to 500 and the minimum number of instances in each leaf during the training of the trees was fixed to 10 . The classification models developed in this work were tested on data not used for training or validation (Table 2). To determine the accuracy of the different algorithms studied the results from the different classification algorithms were compared to the images recorded at the same time as the US measurements.

\section{Results and Discussions}

Figure 3 displays the US energy from the first repeat in the cleaning experiments for different fouling materials and temperatures. The results for gravy at $12{ }^{\circ} \mathrm{C}$ is not shown as it took significantly longer than the other experiments. The results show that as the fouling material is removed from the surface the US energy increases. The results show that the value of US Energy for the clean surface is dependent on the temperature of the experiment. For the experiments at $12{ }^{\circ} \mathrm{C}$ the final value is approximately $1.8 \mathrm{e}^{8}$ whereas for the experiments at $45{ }^{\circ} \mathrm{C}$ the value is approximately $2.1-2.2 \mathrm{e}^{8}$. This result is unsurprising as US wave propagation is known to be highly dependent on temperature. The results in Figure 3 also show that the time taken to remove the fouling from the surface changes for the fouling materials and temperatures studied. The fastest cleaning time was malt at $45{ }^{\circ} \mathrm{C}$ (approximately two minutes) and the slowest cleaning time was gravy at $45{ }^{\circ} \mathrm{C}$ (approximately 12 minutes). For the gravy and malt experiments, which were performed at two different temperatures, the cleaning occurred much faster at the higher temperature. The mean and standard deviation of the cleaning times for the different fouling materials and temperatures are shown in Table 3. Only two experiments were successful at cleaning the gravy at the lower temperature and the time to clean was very different for both experiments. The increase in US energy once the surface is clean is a result of differences in acoustic impedance between the fouling material and the flowing water. The semi-solid fouling material has an acoustic impedance closer to that of the stainless steel wall so the reflection coefficient and US energy of the reflected US wave is lower when the fouling is present. The high values for standard deviation presented in Table 3 are due to variability in the application of the fouling layer between repeat experiments and natural variability of the cleaning process. Gravy had the 
highest standard deviation as only two repeats were possible with this material due to challenges associated with totally removing the fouling.

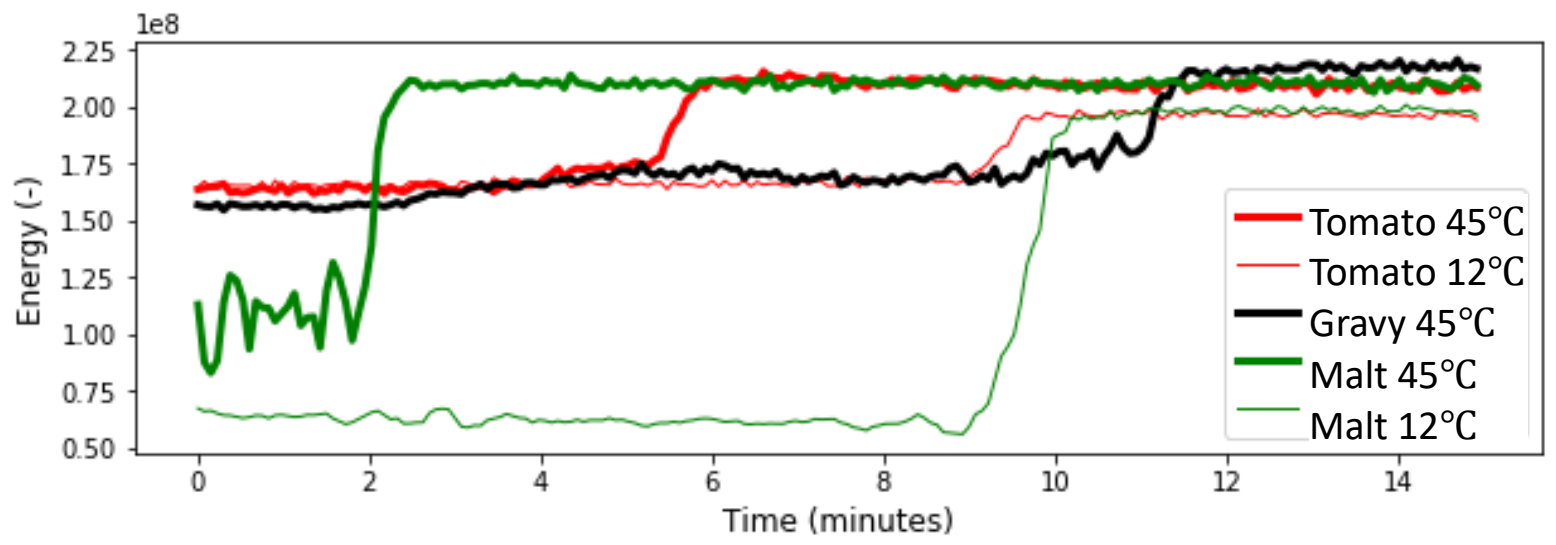

413

Figure 3: Ultrasonic energy of received wave during the cleaning of different fouling materials at low $\left(12{ }^{\circ} \mathrm{C}\right)$ and high temperature $\left(45^{\circ} \mathrm{C}\right)$. Repeat 1 shown for all materials.

Table 3: Mean and standard deviation cleaning times for the different food materials.

\begin{tabular}{|l|c|c|c|c|c|c|}
\hline Material & Tom $12^{\circ} \mathrm{C}$ & Tom $45^{\circ} \mathrm{C}$ & Gravy $12^{\circ} \mathrm{C}$ & Gravy $45^{\circ} \mathrm{C}$ & Malt $12^{\circ} \mathrm{C}$ & Malt $45^{\circ} \mathrm{C}$ \\
\hline Mean cleaning time (s) & 542 & 462 & 4061 & 1087 & 772 & 132 \\
\hline STD cleaning time (s) & 245 & 110 & 2598 & 537 & 76 & 21 \\
\hline
\end{tabular}

Previous research exists studying the cleaning mechanism of different fouling material. It has been proposed that the principle cleaning mechanism is either fluid mechanical removal or diffusion reaction removal (Fryer and Asteriadou, 2009). The experiments reported in this work indicate that the tomato and gravy were cleaned via fluid mechanical removal whereas the malt was cleaned by diffusion reaction removal. In addition it was observed that the gravy swelled with fluid during the initial stages of cleaning. This phenomena was also observed in other works for different fouling material (Xin et al., 2004).

\subsection{Input data point selection and grid search and K-Fold validation}

Figure 4 (a) shows a received US waveform recorded during the experiments. The non-saturated part of the waveform is coloured black and is located from approximately the $700^{\text {th }}$ data point. The red dots show the 200 selected wave data points used as inputs in the KNN model. These input data points were predominately located between the US wave sampling points 700 to 1250 (Figure 4 (b)). 
a)

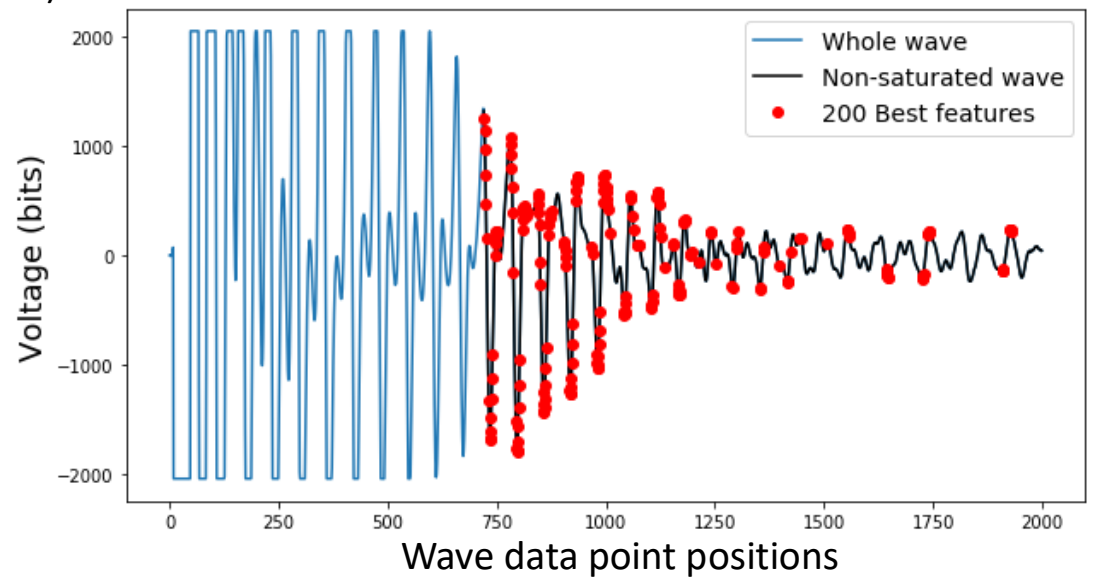

b)

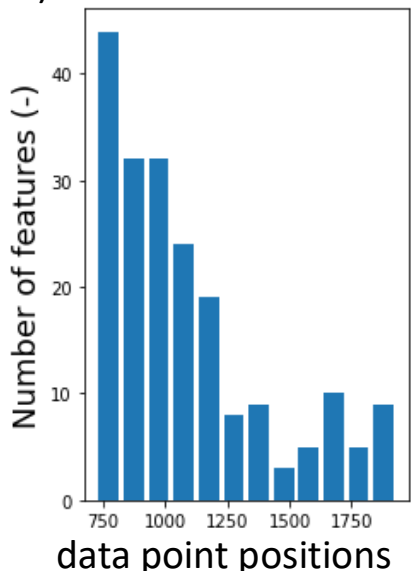

Figure 4. a) Ultrasonic waveform and the 200 best input data points from the wave to be used in the machine learning models. b) Histogram of the positions of the 200 best input data points.

Figure 5 shows how the number of input data points extracted from the US waves affects the validation score for the different classification methods utilised. For all of the different classifiers studied the number of input data points did not have a dramatic effect on the validation score. For consistency the number of input data points utilised was always the value which gave the highest validation score. The optimal number of input data points and the other model parameters utilised can be seen in Table 4.

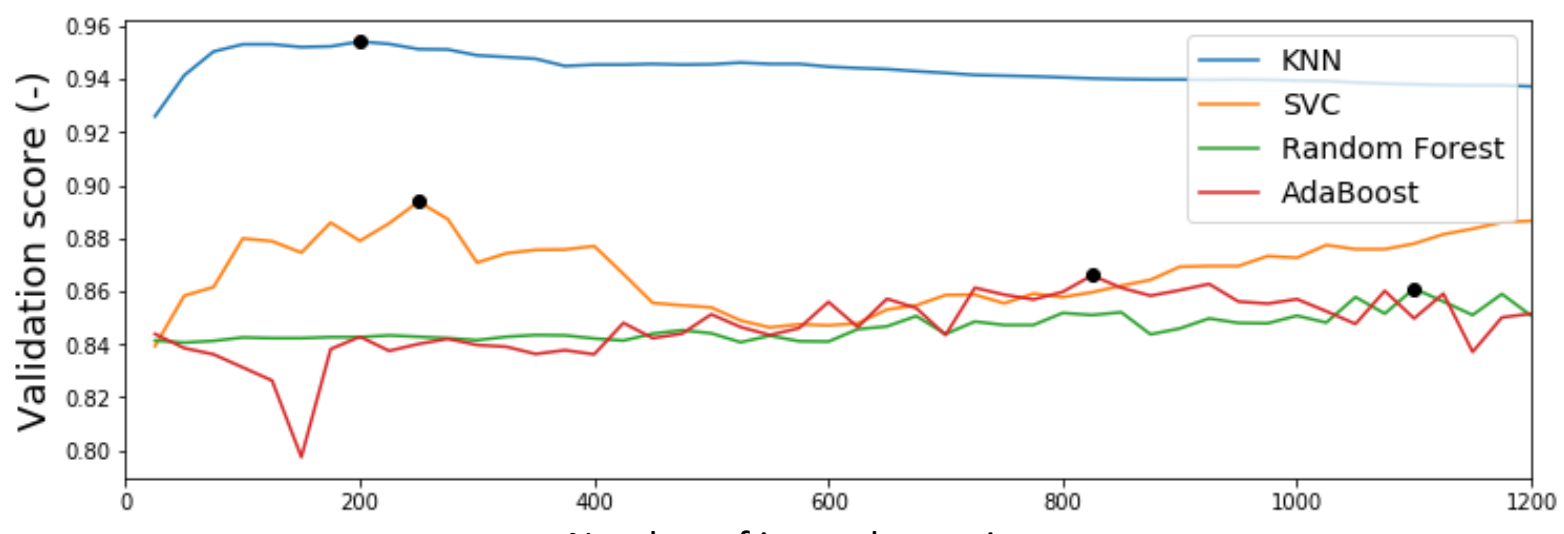

Number of input data points

Figure 5. Validation score of different algorithms as a function of the number of data inputs considered. The maximum score for each algorithm is marked with a black circle.

Table 4: Number of input data points and other parameters used for the ML models. The * represents values chosen manually by the researchers.

\begin{tabular}{|l|l|l|l|l|}
\hline & KNN & SVM & Random Forest & AdaBoost \\
\hline $\begin{array}{l}\text { Number of input data } \\
\text { points: }\end{array}$ & 200 & 250 & 1100 & 825 \\
\hline Number of neighbours: & 105 & - & - & - \\
\hline Number of trees & - & - & $500^{*}$ & $500^{*}$ \\
\hline Maximum depth: & - & - & 4 & $1^{*}$ \\
\hline
\end{tabular}


449

450

451

\begin{tabular}{|l|l|l|l|l|}
\hline $\begin{array}{l}\text { Minimum number of } \\
\text { instances in leafs: }\end{array}$ & - & - & $10^{*}$ & $10^{*}$ \\
\hline Learning rate: & - & - & - & 1 \\
\hline Penalty parameter C: & - & 0.0001 & - & - \\
\hline
\end{tabular}

\subsection{Classification results}

a) Tomato $45^{\circ} \mathrm{C}$

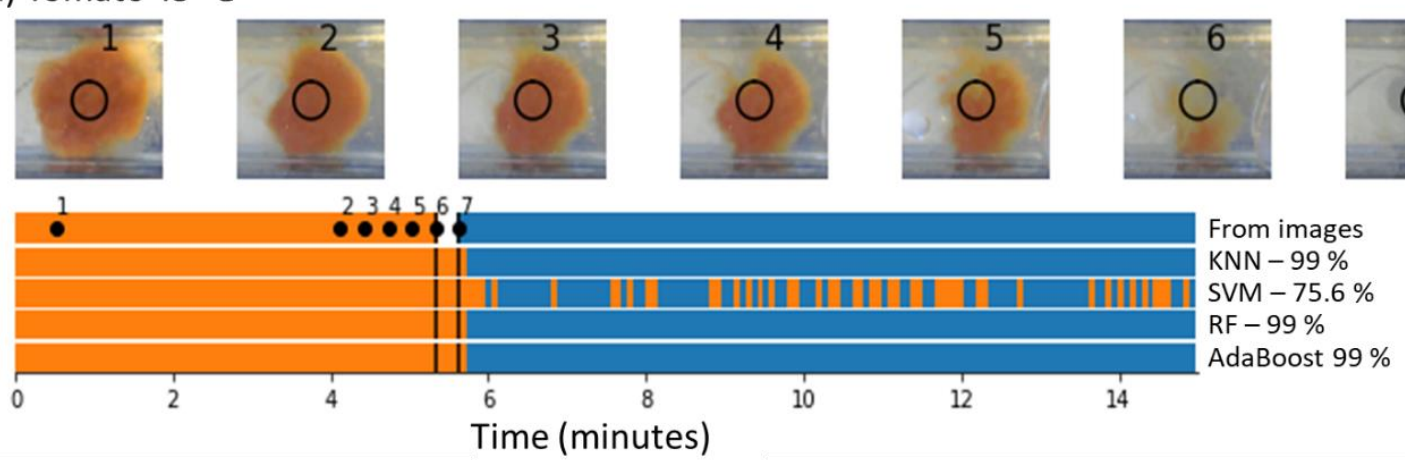

b) Tomato $12{ }^{\circ} \mathrm{C}$

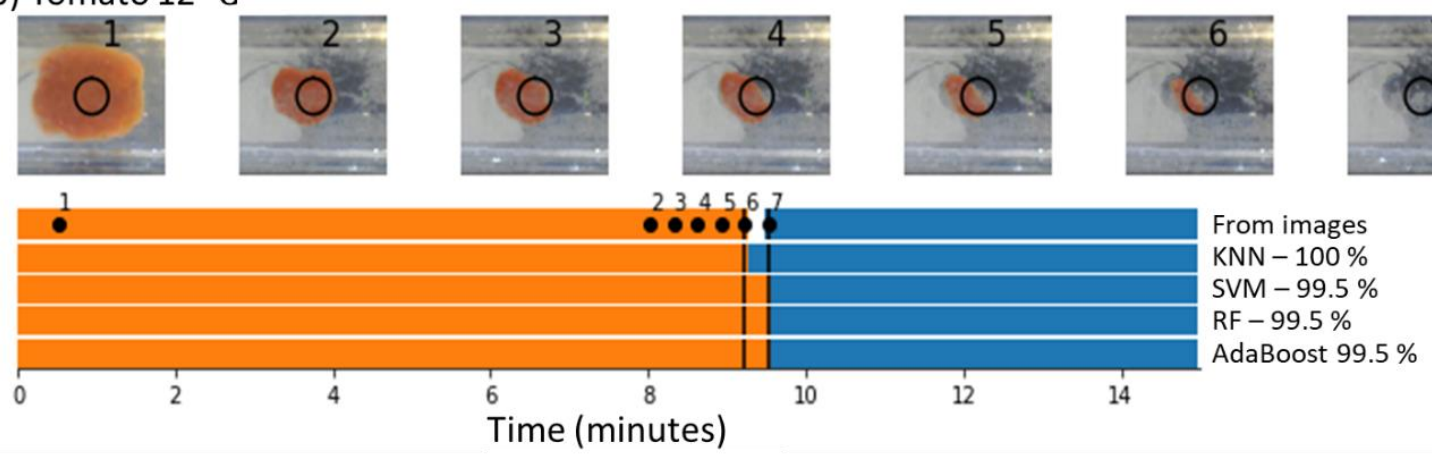

Figure 5. Cleaning predictions of a) tomato at $45{ }^{\circ} \mathrm{C}$ and b) tomato at $12^{\circ} \mathrm{C}$. The colour bars show the cleaning prediction (orange is fouled and blue is clean) at the different image acquisition times. The accuracy of each classification model is shown as a percentage. The images of the fouling include a number to indicate the time on the chart they were recorded.

Figure 5 displays the images of the fouling during cleaning and the results from the ML models for the tomato paste at $45{ }^{\circ} \mathrm{C}$ and $12{ }^{\circ} \mathrm{C}$. The black circles on the images indicates the location of the US transducer, attached to the opposite side of the plate from the fouling. The recorded US signals were labelled fouled if any fouling was present within the circle or clean if no fouling was present in the circle. For the results at the temperature of $12{ }^{\circ} \mathrm{C}$ all ML models performed well with a classification accuracy of $99 \%$ or higher. The predictions for the tomato experiments at $45^{\circ} \mathrm{C}$ all had good prediction accuracy (99\% or higher) except for the SVM. The results for the SVM model often gave predictions that the surface was fouled when it was actually clean. This would result in an autonomous cleaning system, which utilised the sensor measurements, continuing to clean even though the surface fouling had been removed.

The classification results in the current work are comparable to previous research which has used US measurements and $\mathrm{ML}$ to detect fouling and monitoring cleaning processes. Previously reports 
classification values as high as 98\% (Wallhäußer et al., 2011), 100\% (Wallhäußer et al., 2013), 94 \% (Wallhäußer et al., 2014) and 985 (Úbeda et al., 2016). There has been little research which has compared the performance of different classification algorithms for monitoring cleaning using US measurements. However, the previous research has shown that of the different classification methods studied SVM performed better than ANN (Wallhäußer et al., 2013)(Wallhäußer et al., 2014). It should be noted though that this previous work only compared the performance of SVMs to ANNs and studied dairy fouling, which is different to the material used in this work. Artificial Neural Networks were not studied in the current work, so it is difficult to make direct comparisons to this previous research. However, it does highlight that the methodology of combining US measurements and ML is successful for a range of different classification methods and fouling materials increasing the industrial applicability of the method.

a) Gravy $45^{\circ} \mathrm{C}$

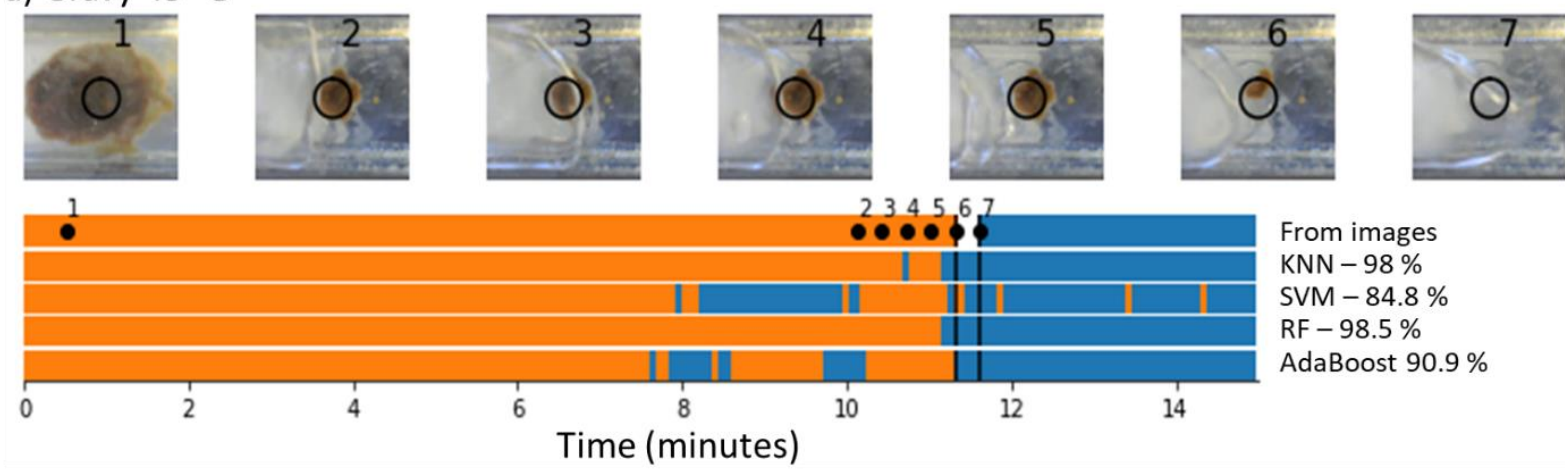

Figure 6. Cleaning predictions of gravy at $45^{\circ} \mathrm{C}$. The colour bars show the cleaning prediction (orange is fouled and blue is clean) at the different image acquisition times. The accuracy of each classification model is shown as a percentage. The images of the fouling include a number to indicate the time on the chart they were recorded.

The classification results for gravy (Figure 6) had much lower performance than for the tomato paste. It is known that different materials foul and are cleaned from surfaces differently and this may be what caused this lower performance. All classifiers except SVM still had a prediction accuracy above $90 \%$, with KNN and RF having the highest accuracy of $98 \%$ and $98.5 \%$ respectively. For this material it was interesting to note that the wrong predictions were often during the cleaning stage where the algorithms predicted the material had been removed when it had not. This could be a problem for an industrial system as a prediction of clean would result in fouling remaining and an unhygienic factory. This raises an important issue when using techniques such as $\mathrm{ML}$ is real-life applications, and the consequences of the predictions should always be considered. An issue that was unique to gravy is that initially during cleaning it swelled with fluid, then became partially detached from the wall before been removed as a single lump. As it became partially detached it is possible that the area opposite 
a) Malt $45^{\circ} \mathrm{C}$

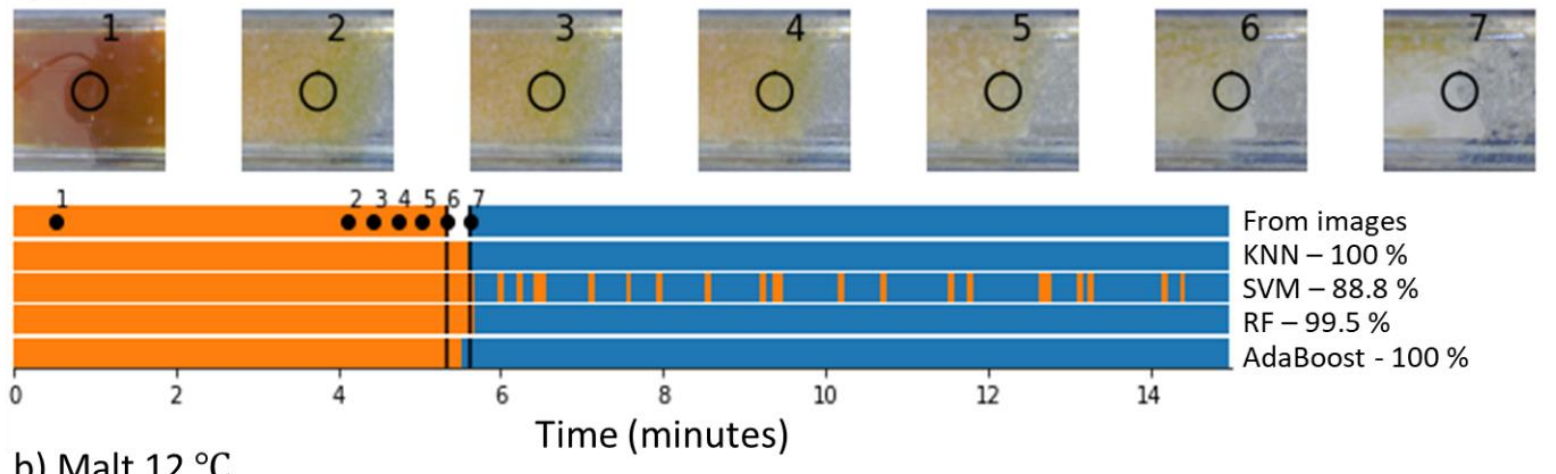

501

502

503

504

505

506

507

508

509

510

511

512

513

514

515

516
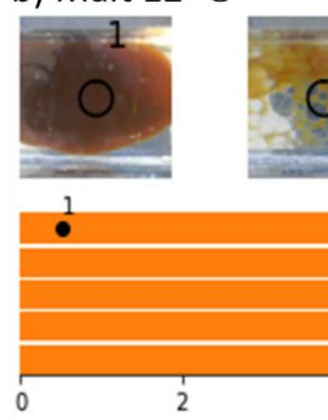

Time (minutes)

From images KNN - $100 \%$ RF - 99.5\% 4
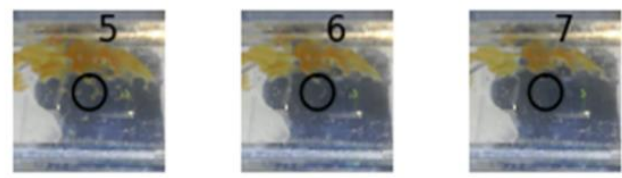

From images KNN $-98.5 \%$ SVM $-97.5 \%$ RF $-100 \%$ AdaBoost $100 \%$

Time (minutes)

Figure 7. Cleaning predictions of a) malt at $45^{\circ} \mathrm{C}$ and b) malt at $12{ }^{\circ} \mathrm{C}$. The colour bars show the cleaning prediction (orange is fouled and blue is clean) at the different image acquisition times. The accuracy of each classification model is shown as a percentage. The images of the fouling include a number to indicate the time on the chart they were recorded.

The experiments for the cleaning of malt had the highest prediction accuracy of all materials studied (Figure 7). These predictions were $97.5 \%$ or higher for all of the classifiers except the SVM. As was the case for the tomato fouling the SVM performed much better for the experiments at $12{ }^{\circ} \mathrm{C}$ than $45^{\circ} \mathrm{C}$. The images of the cleaning for malt showed that it was a much more controlled cleaning process. The malt dissolved into the fluid rather than been removed by the mechanical motion, as was the case with the tomato paste and gravy. It is known that different types of materials clean differently (Fryer and Asteriadou, 2009) and it may be the case that materials that dissolve during cleaning are much more suitable for monitoring using US measurements and classification algorithms. Table 5 includes all of the algorithms accuracy for the different materials and temperatures. As discussed previously SVM was the lowest performing algorithm whereas in general KNN and Adaboost performed better. 
522 Table 5. Performance of the different machine learning methods for different fouling materials and 523 temperatures.

\begin{tabular}{|c|c|c|c|c|c|c|}
\hline \multirow{2}{*}{ Material } & \multirow[b]{2}{*}{ Temperature } & \multirow[b]{2}{*}{ Run } & \multicolumn{4}{|c|}{ Machine Learning Method } \\
\hline & & & KNN & SVM & $\mathbf{R F}$ & Adaboost \\
\hline \multirow{4}{*}{ Tomato } & \multirow{2}{*}{ Hot $45^{\circ} \mathrm{C}$} & 1 & 99 & 72.1 & 99 & 99 \\
\hline & & 2 & 100 & 54.3 & 97 & 97.5 \\
\hline & \multirow{2}{*}{$12{ }^{\circ} \mathrm{C}$} & 1 & 100 & 99.5 & 99.5 & 100 \\
\hline & & 2 & 100 & 99 & 99.5 & 99 \\
\hline \multirow{2}{*}{ Gravy } & \multirow{2}{*}{ Hot $45^{\circ} \mathrm{C}$} & 1 & 97.5 & 84.3 & 99 & 95.4 \\
\hline & & 2 & 98 & 77.7 & 99.5 & 99.5 \\
\hline \multirow{4}{*}{ Malt } & \multirow{2}{*}{ Hot $45^{\circ} \mathrm{C}$} & 1 & 100 & 80.7 & 100 & 100 \\
\hline & & 2 & 100 & 87.3 & 99.5 & 100 \\
\hline & \multirow{2}{*}{$12^{\circ} \mathrm{C}$} & 1 & 100 & 99.5 & 99.5 & 100 \\
\hline & & 2 & 98.5 & 97.5 & 100 & 99.5 \\
\hline \multicolumn{3}{|c|}{ TOTAL } & 99.3 & 85.19 & 99.25 & 98.99 \\
\hline
\end{tabular}

\subsubsection{Physical features}

Table 6 shows the results of the different ML methods when trained using the US energy as the input.

527 For malt the results were comparable to those where input data points were extracted from the

528 recorded US waves. However, for tomato and gravy the prediction accuracy was much lower with 529 values in the 70s, 80s and low 90s percent. As was the case with the results using input data points 530 from the US waveforms the SVM models were the worst performing, with the three other methods 531 having comparable performance. These results indicate that extracting input data points directly from 532 the US waves is a more suitable method than using physical features.

533 Table 6: Performance of the different classification methods using US energy as the input for the 534 models.

\begin{tabular}{|c|c|c|c|c|c|c|}
\hline & \multicolumn{4}{|c|}{ Machine Learning Method } \\
\hline Material & Temperature & Run & KN & SVM & RF & AD \\
\hline \multirow{4}{*}{ Tomato } & \multirow{2}{*}{$45^{\circ} \mathrm{C}$} & 1 & 85.3 & 76.6 & 79.2 & 81.7 \\
\hline & & 2 & 84.8 & 79.2 & 82.2 & 83.8 \\
\hline & \multirow{2}{*}{$12^{\circ} \mathrm{C}$} & 1 & 70.1 & 42.1 & 57.9 & 68 \\
\hline & & 2 & 92.4 & 67.5 & 83.8 & 91.9 \\
\hline \multirow{2}{*}{ Gravy } & \multirow{2}{*}{$45^{\circ} \mathrm{C}$} & 1 & 91.4 & 78.7 & 83.2 & 88.3 \\
\hline & & 2 & 94.9 & 94.9 & 94.4 & 93.9 \\
\hline \multirow{2}{*}{ Malt } & \multirow{2}{*}{$45^{\circ} \mathrm{C}$} & 1 & 100 & 100 & 100 & 99.5 \\
\hline & & 2 & 100 & 100 & 100 & 99.5 \\
\hline
\end{tabular}




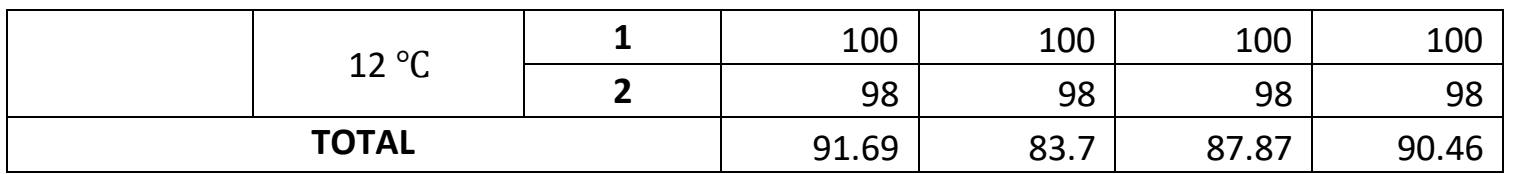

535

536

537

538

539

540

541

542

543

544

545

546

547

548

549

550

551

552

553

554

555

556

557

558

559

560

Previous research has studied the most suitable method for extracting input data features from US waves for classification models. This previous research was not focussed on monitoring cleaning processes but assessing the quality of welded joints (Cruz et al., 2017) and laminated materials (Simas Filho et al., 2016). These works studied a range of different signal processing methods in addition to using the full US waves and principle components calculated from the full waves. These works showed that the highest classification performance was achieved when input data features are extracted from the full waves, which is consistent with our findings. It should be noted though that they did not utilise sample points taken directly from the waves as presented in this current work (Section 2.3.1.1.) but used Principal Component Analysis (PCA) to calculate a new set of features to be used in the models.

\subsubsection{Effect of training data set}

An issue when using ML techniques is having a data set of sufficient size which fully represents the system under investigation. It is important to understand how training data sets should be developed and what data can be grouped together or where training needs to be performed separately. In this section the use of different data sets on the prediction accuracy of the ML Models was studied. For the results presented in Figures 5 to 7 and Tables 5 and 6 data extracted from a total of 7800 US waveforms were used to train the algorithms. This data represented US measurements from all three different fouling materials at the two temperatures studied. Tables 7-9 show the classification accuracy for the different ML models and fouling materials where they were trained and tested individually for each material.

Table 7: Performance of the different machine learning for tomato paste. The models were trained only using the tomato paste data.

\begin{tabular}{|l|c|c|c|c|c|c|}
\cline { 3 - 7 } \multicolumn{2}{c|}{} & \multicolumn{5}{c|}{ Algorithm } \\
\hline \multirow{4}{*}{ Material } & Temperature & Run & KNN & SVM & RF & Adaboost \\
\cline { 3 - 7 } & \multirow{2}{*}{$45^{\circ} \mathrm{C}$} & $\mathbf{1}$ & 36.5 & 36.5 & 36.5 & 36.5 \\
\cline { 3 - 7 } & & $\mathbf{2}$ & 30.5 & 30.5 & 30.5 & 30.5 \\
\cline { 3 - 7 } & \multirow{2}{*}{$12{ }^{\circ} \mathrm{C}$} & $\mathbf{1}$ & 100 & 99.5 & 99.5 & 99.5 \\
\cline { 3 - 7 } & & $\mathbf{2}$ & 99 & 99.5 & 100 & 99 \\
\hline \multicolumn{3}{|c|}{ TOTAL } & 66.5 & 66.5 & 66.625 & 66.375 \\
\hline
\end{tabular}

Table 8: Performance of the different machine learning models for gravy. The models were trained only using the gravy data.

\begin{tabular}{|c|c|c|c|c|c|c|}
\cline { 4 - 7 } \multicolumn{2}{c|}{} & \multicolumn{5}{c|}{ Algorithm } \\
\hline Material & Temperature & Run & KN & SVM & RF & Adaboost \\
\hline Gravy & $45^{\circ} \mathrm{C}$ & $\mathbf{1}$ & 77.2 & 99 & 77.2 & 89.3 \\
\hline
\end{tabular}




\begin{tabular}{|l|l|l|l|l|l|l|}
\hline & & $\mathbf{2}$ & 46.7 & 96.4 & 44.7 & 58.9 \\
\hline \multicolumn{2}{|c|}{ TOTAL } & 61.95 & 97.7 & 60.9 & 74.1 \\
\hline
\end{tabular}

561

562

563

564

565

566

567

568

569

570

571

572

573

574

575

576

577

578

579

580

581

582

583

584

585

586

587

588

589

Table 9: Performance of the different machine learning models for malt extract. The models were trained only using the malt extract data.

\begin{tabular}{|c|c|c|c|c|c|c|}
\hline \multirow{2}{*}{ Material } & \multirow{2}{*}{ Temperature } & \multirow[b]{2}{*}{ Run } & \multicolumn{4}{|c|}{ Algorithm } \\
\hline & & & KN & SVM & RF & Adaboost \\
\hline \multirow{4}{*}{ Malt } & \multirow{2}{*}{$45^{\circ} \mathrm{C}$} & 1 & 99.5 & 99.5 & 100 & 100 \\
\hline & & 2 & 100 & 100 & 100 & 100 \\
\hline & \multirow{2}{*}{$12^{\circ} \mathrm{C}$} & 1 & 100 & 96.4 & 96.4 & 96.4 \\
\hline & & 2 & 97 & 98 & 93.4 & 97 \\
\hline \multicolumn{3}{|c|}{ TOTAL } & 99.1 & 98.5 & 97.5 & 98.4 \\
\hline
\end{tabular}

In general the classification accuracy was higher when training was performed for all materials together (Table 5) than for the individual materials separately (Tables 7-9). This is most likely due to the larger training set used to develop those classifiers. In the instance of this report it is acceptable to combine the data as the final condition of the test section (clean) is the same regardless of the fouling material. Although the different fouling materials had different effects on the US wave reflection properties and cleaned via different mechanisma the final result of a clean test section should result in a similar reflected wave. Another reason that the larger data set is more suitable is that it covers a larger range of temperature variations. US wave propagation in known to be extremely sensitive to temperature and although the experiments were performed at two temperatures small temperature changes $\left(\sim 1-2^{\circ} \mathrm{C}\right)$ would occur between repeats at each temperature due to variations in ambient laboratory conditions during data collection. The larger data set would have more data at different temperatures so should be able to account for these affects during classification.

The current work uses a single transducer which is only capable of detecting fouling in a single location. For the industrial application of this technology multiple US transducers would be utilised. These would be placed at the locations in the processing equipment known to be the most difficult to clean. The addition advantage of using multiple transducers is that they would increase the volume of data collected and improve the prediction capabilities of the ML algorithms.

\section{Conclusions}

This work has studied the use of US measurements and ML classification methods to predict when fouling has been removed from a stainless steel test section, for a range of different food fouling materials. It was found that the different materials all cleaned differently from the surface of the test section and could all be monitored using the US measurements and ML models. A range of different classification methods were studied and it was found that KNN, RF and Adaboost all performed well. Classification accuracy was highest for the malt material which had the most repeatable and controlled cleaning. For any ML approach it is essential to have the most suitable input data, and this 
590

591

592

593

594

595

596

597

598

599

600

601

602

603

604

605

606

607

608

609

610

611

612

613

614

615

616

617

618

619

620

621

622

623

624

625

626

627

628

629

630

631

632

work has shown that the models performed better when input data points were extracted directly from the recorded US waves, than using physical features. The results also showed the importance of a large training data set and that the models performed better when training data used all of the different materials than only for the fouling material testing was completed for. It is believed this is because the final state of a clean test section was similar regardless of the fouling material and the larger training set would account for more variation of temperature experienced during the cleaning. This work has shown that the combination of US measurements and ML techniques can be used to determine when fouling has been removed from a surface and therefore a valuable method for optimising CIP processes.

Funding

This work was supported by the Innovate UK projects 103936 and 132205.

\section{References}

Al-Aufi, Y.A., Hewakandamby, B.N., Dimitrakis, G., Holmes, M., Hasan, A., Watson, N.J., 2019. Thin film thickness measurements in two phase annular flows using ultrasonic pulse echo techniques. Flow Meas. Instrum. 66, 67-78. https://doi.org/10.1016/J.FLOWMEASINST.2019.02.008

Alpaydin, E., 2014. Introduction to Machine Learning Ethem Alpaydin., Introduction to machine learning.

Berg, T.H.A., Ottosen, N., Berg, F. Van Den, Ipsen, R., 2017. LWT - Food Science and Technology Inline UV-Vis spectroscopy to monitor and optimize cleaning-in-place ( CIP ) of whey fi ltration plants. LWT - Food Sci. Technol. 75, 164-170. https://doi.org/10.1016/j.Iwt.2016.08.014

Boser, B.E., Guyon, I.M., Vapnik, V.N., 2004. A training algorithm for optimal margin classifiers. https://doi.org/10.1145/130385.130401

Caruana, R., 1997. Multitask Learning. Mach. Learn. https://doi.org/10.1023/A:1007379606734

Cau, F., Fanni, A., Montisci, A., Testoni, P., Usai, M., 2005. Artificial neural networks for nondestructive evaluation with ultrasonic waves in not accessible. Conf. Rec. - IAS Annu. Meet. (IEEE Ind. Appl. Soc. 1, 685-692. https://doi.org/10.1109/IAS.2005.1518382

Chen, B., Callens, D., Campistron, P., Moulin, E., Debreyne, P., Delaplace, G., 2019. Monitoring cleaning cycles of fouled ducts using ultrasonic coda wave interferometry (CWI). Ultrasonics 01. https://doi.org/10.1016/j.ultras.2018.12.011

Chen, X.D., Li, D.X.Y., Lin, S.X.Q., Necati, O., 2003. On-line fouling / cleaning detection by measuring electric resistance -- equipment development and application to milk fouling detection and chemical cleaning monitoring. Science (80-. ). https://doi.org/10.1016/S0260-8774(03)00085-2

Cheong, Y.M., Kim, K.M., Kim, D.J., 2017. High-temperature ultrasonic thickness monitoring for pipe thinning in a flow-accelerated corrosion proof test facility. Nucl. Eng. Technol. 49, 1463-1471. https://doi.org/10.1016/j.net.2017.05.002

Cruz, F.C., Simas Filho, E.F., Albuquerque, M.C.S., Silva, I.C., Farias, C.T.T., Gouvêa, L.L., 2017. Efficient feature selection for neural network based detection of flaws in steel welded joints using ultrasound testing. Ultrasonics 73, 1-8. https://doi.org/10.1016/j.ultras.2016.08.017

Cuckston, G.L., Alam, Z., Goodwin, J., Ward, G., Wilson, D.I., 2019. Quantifying the e ff ect of solution formulation on the removal of soft solid food deposits from stainless steel substrates. J. Food 
Eng. 243, 22-32. https://doi.org/10.1016/j.jfoodeng.2018.08.018

Eide, M.H., Homleid, J.P., Mattsson, B., 2003. Life cycle assessment (LCA) of cleaning-in-place processes in dairies. LWT - Food Sci. Technol. 36, 303-314. https://doi.org/10.1016/S00236438(02)00211-6

Escrig, J., Woolley, E., Rangappa, S., Simeone, A., Watson, N.J., 2019. Clean-in-place monitoring of different food fouling materials using ultrasonic measurements. Food Control 104, 358-366. https://doi.org/10.1016/J.FOODCONT.2019.05.013

Fan, M., Phinney, D.M., Heldman, D.R., 2018. The impact of clean-in-place parameters on rinse water effectiveness and ef fi ciency. J. Food Eng. 222, 276-283. https://doi.org/10.1016/j.jfoodeng.2017.11.029

Figueiredo, M.M.F., Goncalves, J.L., Nakashima, A.M.V., Fileti, A.M.F., Carvalho, R.D.M., 2016. The use of an ultrasonic technique and neural networks for identification of the flow pattern and measurement of the gas volume fraction in multiphase flows. Exp. Therm. Fluid Sci. 70, 29-50. https://doi.org/10.1016/j.expthermflusci.2015.08.010

Fratamico, P.M., Annous, B.A., Guenther, N.W., 2009. Biofilms in the food and beverage industries. Biofilms Food Beverage Ind. 1-580. https://doi.org/10.1533/9781845697167

Fryer, P.J., Asteriadou, K., 2009. A prototype cleaning map : A classification of industrial cleaning processes. Trends Food Sci. Technol. 20, 255-262. https://doi.org/10.1016/j.tifs.2009.03.005

Fryer, P.J., Christian, G.K., Liu, W., 2006. How hygiene happens: Physics and chemistry of cleaning. Int. J. Dairy Technol. 59, 76-84. https://doi.org/10.1111/j.1471-0307.2006.00249.x

Garcia, R.F., 2012. Improving heat exchanger supervision using neural networks and rule based techniques. Expert Syst. Appl. 39, 3012-3021. https://doi.org/10.1016/j.eswa.2011.08.163

Goodfellow, I., Bengio, Y., Courville, A., 2016. Deep Learning. MIT Press.

Guérin, R., Ronse, G., Bouvier, L., Debreyne, P., Delaplace, G., 2007. Structure and rate of growth of whey protein deposit from in situ electrical conductivity during fouling in a plate heat exchanger. Chem. Eng. Sci. 62, 1948-1957. https://doi.org/10.1016/j.ces.2006.12.038

Hastie, T., Tibshirani, R., Friedman, J., 2009. The Elements of Statistical Learning, Bayesian Forecasting and Dynamic Models. https://doi.org/10.1007/b94608

Lyndgaard, C.B., Rasmussen, M.A., Engelsen, S.B., Thaysen, D., Van Den Berg, F., 2014. Moving from recipe-driven to measurement-based cleaning procedures: Monitoring the Cleaning-In-Place process of whey filtration units by ultraviolet spectroscopy and chemometrics. J. Food Eng. 126, 82-88. https://doi.org/10.1016/j.jfoodeng.2013.10.037

Mohanty, D.K., Singru, P.M., 2014. Fouling analysis of a shell and tube heat exchanger using local linear wavelet neural network. Int. J. Heat Mass Transf. 77, 946-955. https://doi.org/10.1016/j.ijheatmasstransfer.2014.06.007

Park, C., Kim, Y., Park, Y., Kim, S.B., 2018. Multitask learning for virtual metrology in semiconductor manufacturing systems. Comput. Ind. Eng. https://doi.org/10.1016/j.cie.2018.06.024

Pereira, A., Mendes, J., Melo, L.F., 2009. Monitoring cleaning-in-place of shampoo films using nanovibration technology. Sensors Actuators, B Chem. 136, 376-382. https://doi.org/10.1016/j.snb.2008.11.043

Pettigrew, L., Blomenhofer, V., Hubert, S., Groß, F., Delgado, A., 2015. Optimisation of water usage in a brewery clean-in-place system using reference nets. J. Clean. Prod. 87, 583-593. https://doi.org/10.1016/j.jclepro.2014.10.072

Ramsundar, B., Liu, B., Wu, Z., Verras, A., Tudor, M., Sheridan, R.P., Pande, V., 2017. Is Multitask Deep Learning Practical for Pharma? J. Chem. Inf. Model. https://doi.org/10.1021/acs.jcim.7b00146

Riverol, C., Napolitano, V., 2005. Estimation of fouling in a plate heat exchanger through the application on neural networks. J. Chem. Technol. Biotechnol. https://doi.org/10.1002/jctb.1198

Ruppert, D., 2004. The Elements of Statistical Learning: Data Mining, Inference, and Prediction. J. Am. Stat. Assoc. https://doi.org/10.1198/jasa.2004.s339 
684

685

686

687

688

689

690

691

692

693

694

695

696

697

698

699

700

701

702

703

704

705

706

707

708

709

710

711

712

713

714

715

716

717

718

719

720

721

722

723

724

725

726

727

728

729

730

731

732

733

734
Sabzevari, M., Martínez-Muñoz, G., Suárez, A., 2018. Vote-boosting ensembles. Pattern Recognit. https://doi.org/10.1016/j.patcog.2018.05.022

Shetty, G.R., Chellam, S., 2003. Predicting membrane fouling during municipal drinking water nanofiltration using artificial neural networks. J. Memb. Sci. 217, 69-86. https://doi.org/10.1016/S0376-7388(03)00075-9

Simas Filho, E.F., Silva, M.M., Farias, P.C.M.A., Albuquerque, M.C.S., Silva, I.C., Farias, C.T.T., 2016. Flexible decision support system for ultrasound evaluation of fiber-metal laminates implemented in a DSP. NDT E Int. https://doi.org/10.1016/j.ndteint.2015.12.001

Simeone, A., Deng, B., Watson, N., Woolley, E., 2018. Enhanced Clean-In-Place Monitoring Using Ultraviolet Induced Fluorescence and Neural Networks c. https://doi.org/10.3390/s18113742

Simeone, A., Watson, N., Sterritt, I., Woolley, E., 2016. A Multi-sensor Approach for Fouling Level Assessment in Clean-in-place Processes. Procedia CIRP 55, 134-139. https://doi.org/10.1016/J.PROCIR.2016.07.023

Sinner, H., 1959. The Sinner Circle "TACT". In Sinner's Cleaning Philosophy. Henkel.

Sun, L., Zhang, Y., Zheng, X., Yang, S., Qin, Y., 2008. Research on the fouling prediction of heat exchanger based on support vector machine. Proc. - Int. Conf. Intell. Comput. Technol. Autom. ICICTA 2008 1, 240-244. https://doi.org/10.1109/ICICTA.2008.156

Tamachkiarow, A., Flemming, H.C., 2003. On-line monitoring of biofilm formation in a brewery water pipeline system with a fibre optical device. Water Sci. Technol. 47, 19-24. https://doi.org/10.2166/wst.2003.0270

Tlili, M.M., Rousseau, P., Ben Amor, M., Gabrielli, C., 2008. An electrochemical method to study scaling by calcium sulphate of a heat transfer surface. Chem. Eng. Sci. 63, 559-566. https://doi.org/10.1016/j.ces.2007.09.035

Úbeda, M.A., Hussein, W.B., Hussein, M.A., Hinrichs, J., Becker, T.M., 2016. Acoustic sensing and signal processing techniques for monitoring milk fouling cleaning operations. Eng. Life Sci. 16, 67-77. https://doi.org/10.1002/elsc.201400235

Van Asselt, A.J., Van Houwelingen, G., Te Giffel, M.C., 2002. Monitoring system for improving cleaning efficiency of cleaning-in-place processes in dairy environments. Food Bioprod. Process. Trans. Inst. Chem. Eng. Part C 80, 276-280. https://doi.org/10.1205/096030802321154772

Vapnik, V.N., 1999. An overview of statistical learning theory. IEEE Trans. Neural Networks. https://doi.org/10.1109/72.788640

Vieira, M.J., Melo, L.F., Pinheiro, M.M., 1993. Biofilm formation: Hydrodynamic effects on internal diffusion and structure. Biofouling 7, 67-80. https://doi.org/10.1080/08927019309386244

Wallhäußer, E., Hussein, M.A., Becker, T., 2012a. Detection methods of fouling in heat exchangers in the food industry. Food Control 27, 1-10. https://doi.org/10.1016/j.foodcont.2012.02.033

Wallhäußer, E., Hussein, M.A., Becker, T., 2012b. Investigating and understanding fouling in a planar setup using ultrasonic methods. Rev. Sci. Instrum. 83. https://doi.org/10.1063/1.4753992

Wallhäußer, E., Hussein, W.B., Hussein, M.A., Hinrichs, J., Becker, T., 2013. Detection of dairy fouling: Combining ultrasonic measurements and classification methods. Eng. Life Sci. 13, 292-301. https://doi.org/10.1002/elsc.201200081

Wallhaußer, E., Hussein, W.B., Hussein, M.A., Hinrichs, J., Becker, T.M., 2011. On the usage of acoustic properties combined with an artificial neural network - A new approach of determining precense of dairy fouling. J. Food Eng.

Wallhäußer, E., Hussein, W.B., Hussein, M.A., Hinrichs, J., Becker, T.M., 2011. On the usage of acoustic properties combined with an artificial neural network - A new approach of determining presence of dairy fouling. J. Food Eng. 103, 449-456.

https://doi.org/10.1016/j.jfoodeng.2010.11.015

Wallhäußer, E., Sayed, A., Nöbel, S., Hussein, M.A., Hinrichs, J., Becker, T., 2014. Determination of cleaning end of dairy protein fouling using an online system combining ultrasonic and classification methods 506-515. https://doi.org/10.1007/s11947-012-1041-0

Wang, J., Lv, Z., Liang, Y., Deng, L., Li, Z., 2018. Fouling resistance prediction based on GA-Elman 
neural network for circulating cooling water with electromagnetic anti-fouling treatment. J. Energy Inst. 1-8. https://doi.org/10.1016/j.joei.2018.07.022

Watson, N.J., 2015. Ultrasound tomography. Ind. Tomogr. 235-261. https://doi.org/10.1016/B978-178242-118-4.00009-5

Wen, X., Miao, Q., Wang, J., Ju, Z., 2017. A multi-resolution wavelet neural network approach for fouling resistance forecasting of a plate heat exchanger. Appl. Soft Comput. J. 57, 177-196. https://doi.org/10.1016/j.asoc.2017.03.043

Wilson, D.I., 2018. Fouling during food processing - progress in tackling this inconvenient truth. Curr. Opin. Food Sci. 23, 105-112. https://doi.org/10.1016/j.cofs.2018.10.002

Withers, P., 1994. Ultrasonic sensor for the detection of fouling in UHT processing plants. Food Control 5, 67-72. https://doi.org/10.1016/0956-7135(94)90088-4

Withers, P.M., 1996. Ultrasonic, acoustic and optical techniques for the non-invasive detection of fouling in food processing equipment. Trends Food Sci. Technol. 7, 293-298. https://doi.org/10.1016/0924-2244(96)10031-5

Xin, H., Chen, X.D., Özkan, N., 2004. Removal of a model protein foulant from metal surfaces. AIChE J. 50, 1961-1973. https://doi.org/10.1002/aic.10149

Zhang, J., Morris, A.J., Martin, E.B., Kiparissides, C., 1999. Estimation of impurity and fouling in batch polymerisation reactors through the application of neural networks. Comput. Chem. Eng. 23, 301-314. https://doi.org/10.1016/S0098-1354(98)00275-0 